\title{
RELACIÓN ENTRE NEUROCIENCIA Y PROCESOS DE ENSENNANZA-APRENDIZAJE
}

\author{
Marta García Jiménez \\ Facultad Ciencias de la Educación \\ Universidad de Granada \\ martagj@ugr.es \\ María Fernández Cabezas \\ Facultad Ciencias de la Educación \\ Universidad de Granada
}

Recepción Artículo: 10 febrero 2020

Admisión Evaluación: 4 marzo 2020

Informe Evaluador 1: 13 marzo

Informe Evaluador 2: 17 marzo 2020

Aprobación Publicación: 20 abril 2020

\section{RESUMEN}

Esta revisión trata de conceptualizar y entender el término neurociencia y más concretamente, delimitar el término neurociencia vinculado a la educación, así como la neurociencia cognitiva, analizando diversos artículos en los que se pueden encontrar autores a favor de la aplicación de la neurociencia en el aula y otros autores que se contraponen a dicha idea alegando los posibles problemas que pueden surgir en su implementación en el aula. Para ello se han utilizado las bases de datos: Dialnet, Proquest, ISOC, Taylor\&FrancisOnline y Google escolar. Así como las páginas del Mec y del Colegio Trinitarios. Se han analizado 54 artículos y libros de los cuales 15 son relativos a programas y/o proyectos. Se enfatiza el papel del docente en la implementación de metodologías en el aula vinculadas a la neurociencia, así como factores que influyen en el proceso de aprendizaje del alumno, como son: la plasticidad cerebral y la memoria. Por último, se analizarán 9 programas y/o proyectos sobre todo en el ámbito español, aunque se añaden otros implementados fuera de dicho ámbito ( $\mathrm{n}=10)$. Así se realiza, también, un análisis sobre la importancia de implementar más metodologías y estrategias en el ámbito educativo español, ya que se han encontrado escasos resultados al respecto. Con ello se genera una propuesta de extrapolar a las aulas españolas otros programas y/o proyectos implementados en otros contextos, así como generar nuestros propios programas y/o proyectos, para alcanzar un aprendizaje significativo por parte de nuestro alumnado en los distintos niveles educativos.

Palabras clave: neurociencia; educación; aprendizaje; programas de intervención

\section{ABSTRACT}

This review tries to conceptualize and understand the term neuroscience and more specifically, delimit the term neuroscience linked to education, as well as cognitive neuroscience, analyzing various articles in which 


\section{RELACIÓN ENTRE NEUROCIENCIA Y PROCESOS DE ENSEÑANZA-APRENDIZAJE}

authors can be found in favor of the application of neuroscience in the classroom and other authors who oppose this idea, arguing the posible problems that may arise in its implementation in the classroom. For this, the databases have been used: Dialnet, Proquest, ISO, Taylor\&FrancisOnline and Google School. As well as the webs of the MEC and the Trinitarios School. 54 articles and books have been analyzed, of which 15 are related to programs and/or projects. The role of the teacher in the appliance of methodologies in the classroom linked to neuroscience is emphasized, as well as factors that influence the learning process of the student, such as: brain plasticity and memory. Finally, 9 programs and/or projects will be analyzed, especially in the Spanish area, although other implemented outside this area are added $(n=10)$. This is also an analysis of the importance of implementing more methodologies and strategies in the Spanish Educational area, since few results have been found in this regard. This generates a proposal to extrapolate to the Spanish classrooms other programs and/or projects implemented in other contexts, as well as generate our own programs and/or projects, to achieve a significant learning by our students in the different educational levels.

Keywords: neuroscience; learning; education; programs intervention

\section{ANTECEDENTES}

La Neurociencia es un conjunto de ciencias que tiene como eje central el estudio del sistema nervioso y la interrelación entre actividad cerebral, conducta y aprendizaje del ser humano (Pizano, 2010; Redolar, 2002, citado en Benarós, Lipina, Segretin, Hermida y Colombo, 2010). Por su parte, Escorza (2017) cita autores que pusieron de manifiesto la relevancia que tiene el cerebro a nivel de funcionamiento: Alcmeón de Crotona (S.V a.C.), incidió en la importancia del pensamiento y las sensaciones; y Descartes (596-1650), hizo alusión al control sobre la conducta humana; y a nivel estructural: Ramón y Cajal (1852-1934) destacó las perspectivas: anatómica y fisiológica.

En la década de los '90, la 'década del cerebro', la neurociencia comienza a tener un crecimiento significativo debido a los numerosos avances tecnológicos que se producen (Elgier, 2012) y permiten observar en vivo el funcionamiento del cerebro (Cantó, 2015) desde cómo se percibe el mundo que rodea, orientación y navegación en el espacio, hasta cómo afecta el entorno físico (capacidad cognitiva, resolución de conflictos y estado de ánimo) (Sternberg y Wilson, 2006, citados en Mokhtar, Jiménez, Heppell y Segovia, 2016). La neuroimagen no invasiva permite medir las funciones cerebrales en la realización de una tarea cognitiva (Rodrigo, 2010). Al comprender el cerebro, surge la neurociencia cognitiva y educativa, el profesorado mejora su práctica docente y fomenta el desarrollo integral del alumnado (López-Escribano y Moreno, 2014; Bodero, 2017).

\section{Neurociencia y educación}

El vínculo generado entre la neurociencia y educación presentan dos hechos históricos: un encuentro entre investigadores de distintas disciplinas en 1996 intentó disminuirla 'brecha' existente entre ambos términos; y un trabajo de Bruer (1997, citado en Benarós et al., 2010) introdujo el término 'puente', considerar ambos términos como disciplina y los psicólogos cognitivos son los que están mejor preparados para trabajar el vínculo (Bravo, 2014; Codina, 2014a, 2014b; Bruer, 2008, citado en Marina, 2012).

De este vínculo han ido surgiendo múltiples centros, programas y congresos que lo trabajan, como los centros de Neurociencia en la Educación (Universidad de Cambridge), el de Transferencia de la Neurociencia y el Aprendizaje (Alemania), el de Investigación Educacional e Innovacional (OCDE); Ios programas de Mente, Cerebro y Educación (Universidad de Harvard), y el Laboratorio de Aprendizaje (Dinamarca) (Barrios- Tao, 2016, p.398). En el año 2000 se realizó un congreso internacional sobre plasticidad cerebral y periodos de aprendizaje en Nueva York; en 2001, un congreso en Granada sobre la neurociencia en el aula; en el 2002 publican Understanding the Brain (OCDE), proponen vincular la neurociencia con la educación; en 2004 se crea la International Mind, Brain, and Education Society, en 2010, se celebró el I World Congress of Neuroeducation, en Lima; y en España se celebró en I Congreso Nacional de Neurociencia aplicada a la Educación y el I Congreso de Educación Infantil y Neurociencia (Marina, 2012, p.7; Codina, 2014a, p.27-29; Luque y García, 2017, p. 5; Ministerio de Educación, Cultura y Deporte, 2018). 
En cuanto a autores relevantes Marina (2012) cita algunos de ellos como Burunat y Damas (2002) publican Introducción a la Neuropedagogía; Mora (2007) incorpora educación y neurocultura; Iglesias (2008) publica Bases biológicas de la educación; Ortiz (2009) publica Neurociencia y Educación; Maya y Rivero (2010) publican un informe Conocer el cerebro para la excelencia de la educación; y Nieto (2011) publica Neurodidáctica (p.8).

\section{Neurociencia y aprendizaje. Cerebro, plasticidad cerebral y memoria}

La neurociencia y las teorías cognitivas conforman un referente para entender los procesos de aprendizaje, pudiendo justificar el tipo de metodologías pedagógicas utilizadas en cada situación y el tipo de estrategias que el profesorado debe emplear para mejorar la capacidad de asimilación de conocimientos del alumnado. El proceso de aprendizaje es de carácter cognitivo y bioquímico, las conexiones neuronales transmiten impulsos electroquímicos que permiten al ser humano adaptarse, mediante la denominada 'plasticidad cerebral', al entorno. Dichas conexiones pueden ser redes alternativas o sustitución de nuevas redes actualizadas (Flórez, 1999; Ratey, 2002; Spitzer, 2005; Mora, 2013, citados en Jauset- Berrocal, Martínez y Añaños, 2017).

La plasticidad cerebral es la capacidad de adaptación al entorno que tiene el cerebro y permite la construcción de redes neuronales estables partiendo de experiencias, aprendizajes y estimulaciones (sensoriales y cognitivas) (Aguilar, Espinoza, Oruro y León, 2012, citados en Aguilar et al., 2017). El vínculo que une los pensamientos con la experiencia es la memoria, definida por Aguilar et al. (2017, p. 105) como 'la capacidad de adquirir, retener, almacenar y evocar información del ambiente'. Se distinguen, según Gleichgerrcht (2013, citado en Aguilar et al., 2017) tres procesos básicos en ella: codificación, almacenaje y evocación.

En el ámbito educativo, las tareas tienen que tener un carácter interesante y desafiante a nivel cognitivo que permitirá la adquisición de contenidos debido a su índole novedosa (Yamila, De la Barrera y Travaglia, 2017).

\section{Neurociencia y educación en el aula.}

En el contexto educativo, diversos autores como Piaget, Vygotsky y Ausubel han intentado explicar las conductas de aprendizaje y los procesos mentales desde distintas disciplinas (psicología, sociología, filosofía y antropología) mediante observaciones y evidencias empíricas para generar estrategias de enseñanza basadas en el aprendizaje significativo (Escorza, 2017).

Las etapas educativas más relevantes son Educación Infantil y Primaria, ya que el alumnado empieza a generar habilidades sociales y cognitivas, por lo que el profesorado tiene que investigar sobre su propia práctica y comprobar qué estrategias son más adecuadas en cada momento, así como hacer partícipe al alumnado de su propio proceso de aprendizaje (Escorza, 2017; Luque y García, 2017).

Existen autores como Escorza (2017) que enmarcan los estilos de aprendizaje dentro de 'neuromitos', término creado por Crockard en los '80 y alude a ideas vinculadas al cerebro sin sustentación científica. Y un porcentaje elevado de profesorado cree en la enseñanza a través del estilo VAK (visual, auditivo y kinestésico), sin embargo, se ha demostrado el carácter no lineal de la neurociencia, la información que llega al cerebro se distribuye y codifica, por lo que hay autores que lo corroboran (Krätzig y Arbuthnott, 2006; Coffield, Moseley, Hall y Ecclestone, 2004, citados en Escorza, 2017).

La afirmación de diversos investigadores sobre el interés que parte del profesorado tiene hacia el vínculo neurociencia y educación es patente, y abogan por desarrollar programas que ayuden a comprender el desarrollo evolutivo del cerebro y su funcionamiento, así como divulgar los avances que se realicen al respecto (Pickering y Howard- Jones, 2007, citados en Luque y García ,2017).

\section{Proyectos y programas en España}

En España, se han generado propuestas didácticas que se sustentan en estudios neurodidácticos en el aula, el colegio concertado Monserrat (Barcelona) utiliza metodología de cooperación, aprendizaje por proyectos, entre otros) y el colegio Zola las Rozas (Madrid) utiliza metodología basada en el aprendizaje en los rincones del aula (Guirado, 2017). 


\section{RELACIÓN ENTRE NEUROCIENCIA Y PROCESOS DE ENSEÑANZA-APRENDIZAJE}

El desarrollo de proyectos vinculando neurociencia y educación en el aula se ha ido observando en las titulaciones de Ciencias de la Educación y Psicología, como: el proyecto piloto que realizó Luque (2015) comprendía cuatro grados universitarios y dos másteres, con una metodología de investigación - acción y la realización de diversas actividades entre las que estaba la elaboración de un programa de neuroeducación en el aula, cuya finalidad era la mejora de los aprendizajes del alumnado; y el proyecto en el Grado de Pedagogía de Luque y García (2017), ampliando el conocimiento en contenidos neuroeducativos.

Se han ido generando programas vinculando ambos términos, destacando el pionero que se ha desarrollado en Madrid y engloba a 29 colegios (públicos y concertados), dirigido a la etapa de infantil, con posibilidad de ampliación a otras etapas y la presencia de las familias para concienciarla en cómo la neurociencia favorece el aprendizaje del alumnado (Guirado, 2017; Colegio Trinitarios, 2014).

\section{OBJETIVOS DEL ESTUDIO}

Los objetivos del presente estudio son los siguientes:

Revisar los estudios previos que se han realizado en relación con la neurociencia aplicada a la educación.

Estudiar la relación existente entre la neurociencia y los procesos de enseñanza- aprendizaje.

Revisar diversos estudios que lleven la neurociencia al aula, centrándonos en el ámbito español.

\section{MUESTRA DEL ESTUDIO}

El presente estudio parte de un primer planteamiento sobre el vínculo entre neurociencia y educación en el aula mediante proyectos y programas en el contexto español (nacional), se descubrió la falta de información y se decidió la ampliación para recoger más información a nivel internacional.

Se ha realizado una búsqueda en diversas bases de datos seleccionadas del catálogo digital de la biblioteca de la Universidad de Granada: Dialnet, Proquest, ISOC, Taylor\&FrancisOnline; así como Google escolar. El periodo elegido de búsqueda se ha centrado desde 2009 a 2018. Para las búsquedas realizadas sobre programas se han utilizado las webs del Ministerio de Educación, Cultura y Deporte de España y el Colegio Trinitarios.

\section{METODOLOGÍA DEL ESTUDIO}

La metodología seguida ha sido una búsqueda de literatura en diferentes bases de datos anteriormente mencionadas y relacionando entre sí diversos descriptores de búsqueda, tanto en español como en inglés: 'neurociencia', ' aprendizaje', 'programas', 'proyectos', 'intervención', 'aula', 'interacción', 'neuroeducación', 'neuroeducativos', 'neurodidáctica', 'neuropedagogía', 'psicología cognitiva', 'neurociencia cognitiva', 'neurodiversidad', 'neuromitos', 'procesos enseñanza- aprendizaje', ' plasticidad cerebral', ' learning', 'neuroscience', ' brain', ' memory', ' education' , 'cognition', 'neuropsychology' y ' neurodidactics'.

Se ha depurado un total de 54 documentos, sobre programas y proyectos vinculando neurociencia y educación en el aula (15 documentos), y siete de ellos en España durante el periodo de 2008-2018, el resto ( $n=8$ ) realizados en América del Sur. De los siete artículos que contemplan programas y/o proyectos realizados en España se han encontrado un total de nueve programas y/o proyectos, así mismo de los ocho artículos vinculados a programas y/o proyectos realizados en el extranjero se han encontrado un total de diez programas y/o proyectos.

Del total de artículos revisados un $72.22 \%$ se han utilizado para confeccionar el marco teórico y un $27.78 \%$ corresponde a los artículos con programas y/o proyectos encontrados vinculando neurociencia y educación en el aula. De estos últimos, un 46.67\% corresponde a estudios realizados en España, sin embargo, un 53.33\% corresponde a estudios realizados sobre todo en América del Sur.

\section{DISCUSIÓN DE RESULTADOS ALCANZADOS}

Existen controversias relativas al origen de la neurociencia vinculada a la educación, diversos autores la enmarcan en la década de los '90 (Barrios-Tao, 2016; Elgier, 2012; Cantó, 2015; López-Escribano y Moreno, 2014) y otros autores destacan hitos relevantes previos (Bruer, 1997; Byrnes y Fox, 1998, citados en Benarós et al., 2010). 
Sylvan y Christodoulou (2010, citados en Cantó, 2015) encontraron que los conocimientos sobre la neurociencia estaban cambiando las teorías sobre aprendizaje que había vigentes incorporando estrategias didácticas vinculadas al funcionamiento del cerebro (Wolfe, 2010, citado en Luque, 2015 y en Luque y García, 2017; Twaedosz, 2012, citado en López- Escribano y Moreno, 2014),

La neurociencia cognitiva ha aportado numerosas contribuciones al ámbito educativo, cuyo objeto de estudio son las representaciones mentales que se producen ante determinados procesos de índole cognitivo, emocional, motivacional y psicológico (Atherton y Disket, 2005, citado en Benarós et al., 2010). Cuando el profesorado comprende el funcionamiento del cerebro puede mejorar su práctica educativa y potenciar el desarrollo integral de su alumnado (Bodero, 2017; Carreiras, 2012).

Tras la revisión de los 15 artículos vinculados a los programas y proyectos, se ha contabilizado un total de siete artículos que incluyen nueve implementados en España, Tabla 1. Se destaca que un 55.56\% implementados en 2015, un 33.33\% implementados en 2017 y un 11.11\% implementados en 2016. En cuanto al nivel educativo, se distingue un 33.33\% en el ámbito universitario, un 22.22\% en Educación Infantil, un 11.11\% se implementa tanto en Educación Infantil como en Primaria, un 22.22\% en Secundaria y un 11.11\% no se especifica.

Tabla 1 Programas y Proyectos de neurociencia vinculados a la Educación: nombre, autor/es, año, procedencia y nivel educativo

\begin{tabular}{|c|c|c|c|c|}
\hline Nombre & Autor/es & Año & Procedencia & $\begin{array}{c}\text { Nivel } \\
\text { educativo }\end{array}$ \\
\hline $\begin{array}{l}\text { Neuroeducación. Cerebro } \\
\text { y mente en el aula. } \\
\text { Innovación y mejora de } \\
\text { procesos educativos y de } \\
\text { enseñanza }\end{array}$ & María Jesús Luque & 2015 & España & $\begin{array}{l}\text { Universidad } \\
\text { (Grado y } \\
\text { Máster) }\end{array}$ \\
\hline $\begin{array}{l}\text { Neurociencias en } \\
\text { Ciencias de la Educación. } \\
\text { Coordinación y } \\
\text { formación docente desde } \\
\text { la Universidad }\end{array}$ & $\begin{array}{l}\text { María Jesús Luque } \\
\text { y María del Carmen } \\
\text { García }\end{array}$ & 2017 & España & $\begin{array}{l}\text { Universidad } \\
\text { (Grado de } \\
\text { Pedagogía) }\end{array}$ \\
\hline $\begin{array}{l}\text { Programa de } \\
\text { neurociencia y educación }\end{array}$ & $\begin{array}{l}\text { Consejería de } \\
\text { Educación (CAM) }\end{array}$ & 2015 & España & $\begin{array}{l}\text { Universidad } \\
\text { (Educación } \\
\text { Infantil) }\end{array}$ \\
\hline $\begin{array}{l}\text { Propuestas didácticas en } \\
\text { base a los estudios } \\
\text { neurodidácticos }\end{array}$ & $\begin{array}{l}\text { Colegios } \\
\text { (Monserrat de } \\
\text { Barcelona; Zola las } \\
\text { Rozas, Madrid) }\end{array}$ & 2017 & España & $\begin{array}{l}\text { Educación } \\
\text { Infantil }\end{array}$ \\
\hline Neurodidáctica del juego & No se especifica & 2017 & España & $\begin{array}{l}\text { Educación } \\
\text { Infantil y } \\
\text { Primaria }\end{array}$ \\
\hline $\begin{array}{l}\text { Implementación de la } \\
\text { neurodidáctica en el aula }\end{array}$ & $\begin{array}{l}\text { Linea investigadora } \\
\text { (Universidad de } \\
\text { Valencia) }\end{array}$ & 2015 & España & $\begin{array}{l}\text { Educación } \\
\text { Infantil }\end{array}$ \\
\hline $\begin{array}{l}\text { La influencia del sueño y } \\
\text { horario de las sesiones }\end{array}$ & No se especifica & 2015 & España & $1^{\circ}$ E.S.O \\
\hline $\begin{array}{l}\text { La Efectividad de la } \\
\text { metodología de los } \\
\text { mapas mentales }\end{array}$ & No se especifica & 2015 & España & $1^{\circ}$ E.S.O \\
\hline $\begin{array}{l}\text { Influence of eye } \\
\text { movements, auditory } \\
\text { perception and phonemic } \\
\text { awareness in the Reading } \\
\text { process }\end{array}$ & No se especifica & 2016 & España & $\begin{array}{l}\text { No se } \\
\text { especifica }\end{array}$ \\
\hline
\end{tabular}

International Journal of Developmental and Educational Psychology 


\section{RELACIÓN ENTRE NEUROCIENCIA Y PROCESOS DE ENSEÑANZA-APRENDIZAJE}

Con respecto al tipo de evaluación, Tabla 2, un 33.33\% tienen evaluación inicial, seguimiento y evaluación final. Un 44.44\% no especifica su evaluación, un $11.11 \%$ con evaluación inicial y final, un $11.11 \%$ con evaluación final. Según los instrumentos utilizados, un $22.22 \%$ usan cuestionarios, informes..., un $66.67 \%$ no lo especifican, y un $11.11 \%$ para los que especifican el tipo de instrumento utilizado. En relación con los resultados obtenidos, un $66.67 \%$ no los especifican, un $22.22 \%$ apuntan resultados significativos, y un $11.11 \%$ señalan resultados no significativos.

Tabla 2

Programas y Proyectos de neurociencia vinculados a la Educación: nombre, tipo de evaluación, instrumentos utilizados y resultados obtenidos

\begin{tabular}{|c|c|c|c|}
\hline Nombre & $\begin{array}{c}\text { Tipo de } \\
\text { evaluación }\end{array}$ & $\begin{array}{l}\text { Instrumento } \\
\text { s utilizados }\end{array}$ & $\begin{array}{l}\text { Resultados } \\
\text { obtenidos }\end{array}$ \\
\hline $\begin{array}{l}\text { Neuroeducación. Cerebro y } \\
\text { mente en el aula. Innovación } \\
\text { y mejora de procesos } \\
\text { educativos y de enseñanza. }\end{array}$ & $\begin{array}{l}\text { Valoración inicial y } \\
\text { final } \\
\text { Fase de } \\
\text { seguimiento }\end{array}$ & $\begin{array}{l}\text { Cuestionarios } \\
\text {, informes, } \\
\text { participación, } \\
\text { asistencia... }\end{array}$ & No se especifica \\
\hline $\begin{array}{l}\text { Neurociencias en Ciencias de } \\
\text { la Educación. Coordinación y } \\
\text { formación docente desde la } \\
\text { Universidad }\end{array}$ & $\begin{array}{l}\text { Valoración inicial y } \\
\text { final } \\
\text { Fase de } \\
\text { seguimiento }\end{array}$ & $\begin{array}{l}\text { Cuestionarios } \\
\text {, informes, } \\
\text { participación, } \\
\text { asistencia... }\end{array}$ & No se especifica \\
\hline $\begin{array}{l}\text { Programa de neurociencia y } \\
\text { educación }\end{array}$ & $\begin{array}{l}\text { Evaluación inicial, } \\
\text { durante el proceso } \\
\text { y al final }\end{array}$ & $\begin{array}{l}\text { No se } \\
\text { especifica }\end{array}$ & No se especifica \\
\hline $\begin{array}{l}\text { Propuestas didácticas en base } \\
\text { a los estudios neurodidácticos }\end{array}$ & No se especifica & $\begin{array}{l}\text { No se } \\
\text { especifica }\end{array}$ & No se especifica \\
\hline Neurodidáctica del juego & No se especifica & $\begin{array}{l}\text { No se } \\
\text { especifica }\end{array}$ & No se especifica \\
\hline $\begin{array}{l}\text { Implementación de la } \\
\text { neurodidáctica en el aula }\end{array}$ & No se especifica & $\begin{array}{l}\text { No se } \\
\text { especifica }\end{array}$ & $\begin{array}{l}\text { Caulitativos: Con } \\
\text { la aplicación de } \\
\text { estrategias basadas } \\
\text { en la } \\
\text { neurodidáctica se } \\
\text { mejora el clima del } \\
\text { aula }\end{array}$ \\
\hline $\begin{array}{l}\text { La influencia del sueño y } \\
\text { horario de las sesiones }\end{array}$ & $\begin{array}{l}\text { Se realiza un test al } \\
\text { inicio y al final }\end{array}$ & $\begin{array}{l}\text { Actividad } \\
\text { parcial ( } 30 \\
\text { minutos); } \\
\text { Actividad } \\
\text { final (1 hora) }\end{array}$ & $\begin{array}{l}\text { Significativos } \\
\text { (Actividad parcial } \\
\text { y final) }\end{array}$ \\
\hline $\begin{array}{l}\text { La Efectividad de la } \\
\text { metodología de los mapas } \\
\text { mentales }\end{array}$ & $\begin{array}{l}\text { Se realiza un test al } \\
\text { final }\end{array}$ & $\begin{array}{l}\text { No se } \\
\text { especifica }\end{array}$ & $\begin{array}{l}\text { No existen } \\
\text { resultados } \\
\text { significativos }\end{array}$ \\
\hline $\begin{array}{l}\text { Influence of eye movements, } \\
\text { auditory perception and } \\
\text { phonemic awareness in the } \\
\text { Reading process }\end{array}$ & No se especifica & $\begin{array}{l}\text { No se } \\
\text { especifica }\end{array}$ & No se especifica \\
\hline
\end{tabular}


De acuerdo a los contenidos, Tabla 3, un 55.56\% se refieren a contenidos variados, un $44.44 \%$ no se contemplan. Para las actividades relevantes, un 33.33\% exponen el diseño de un programa de neuroeducación, un $33.33 \%$ se vinculan con el horario escolar, el trabajo en fin de semana con la familia, periodo formativo y seis sesiones de trabajo. Y un 33.33\% para los que no las especifican.

Tabla 3

Programas y Proyectos de neurociencia vinculados a la Educación: nombre, contenidos y actividades relevantes

\begin{tabular}{|c|c|c|}
\hline Nombre & Contenidos & Actividades relevantes \\
\hline $\begin{array}{l}\text { Neuroeducación. Cerebro y } \\
\text { mente en el aula. Innovación y } \\
\text { mejora de procesos educativos y } \\
\text { de enseñanza. }\end{array}$ & $\begin{array}{l}3 \text { Fases: } \\
\text { Fase de diseño, fase } \\
\text { de experimentación } \\
\text { y fase de evaluación. } \\
\text { Proceso de } \\
\text { autoformación }\end{array}$ & $\begin{array}{l}\text { Elaboración de un programa } \\
\text { de neuroeducación para su } \\
\text { aplicación práctica en el aula. }\end{array}$ \\
\hline $\begin{array}{l}\text { Neurociencias en Ciencias de la } \\
\text { Educación. Coordinación y } \\
\text { formación docente desde la } \\
\text { Universidad }\end{array}$ & $\begin{array}{l}\text { Ampliar } \\
\text { conocimiento sobre } \\
\text { contenidos } \\
\text { neuroeducativos, } \\
\text { comprender distintos } \\
\text { mecanismos del } \\
\text { cerebro... }\end{array}$ & $\begin{array}{l}\text { Elaboración de un programa } \\
\text { de neuroeducación para su } \\
\text { aplicación práctica en el aula. } \\
\text { Diseño de talleres y cursos } \\
\text { vinculados en neurociencia y } \\
\text { educación }\end{array}$ \\
\hline $\begin{array}{l}\text { Programa de neurociencia y } \\
\text { educación }\end{array}$ & $\begin{array}{l}\text { Mejorar } \\
\text { capacidades: } \\
\text { atención, } \\
\text { respiración, } \\
\text { hidratación... }\end{array}$ & $\begin{array}{l}\text { En horario escolar: } 3 \text { series de } \\
\text { ejercicios ( } 5 \text { tipos distintos). } \\
\text { Intervalo entre aplicaciones } \\
\text { de tres horas } \\
\text { En fin de semana los mismos } \\
\text { ejercicios con la familia }\end{array}$ \\
\hline $\begin{array}{l}\text { Propuestas didácticas en base a } \\
\text { los estudios neurodidácticos }\end{array}$ & No se especifica & No se especifica \\
\hline Neurodidáctica del juego & $\begin{array}{l}\text { Activación de las } \\
\text { emociones }\end{array}$ & No se especifica \\
\hline $\begin{array}{l}\text { Implementación de la } \\
\text { neurodidáctica en el aula }\end{array}$ & $\begin{array}{l}\text { Estrategias docente } \\
\text { basadas en la } \\
\text { neurodidáctica }\end{array}$ & Periodo formativo \\
\hline $\begin{array}{l}\text { La influencia del sueño y horario } \\
\text { de las sesiones }\end{array}$ & No se especifica & Distribuido en 6 sesiones \\
\hline $\begin{array}{l}\text { La Efectividad de la metodología } \\
\text { de los mapas mentales }\end{array}$ & No se especifica & No se especifica \\
\hline $\begin{array}{l}\text { Influence of eye movements, } \\
\text { auditory perception and } \\
\text { phonemic awareness in the } \\
\text { Reading process }\end{array}$ & No se especifica & $\begin{array}{l}\text { Diseño de un programa } \\
\text { vinculado a la neurpsicología } \\
\text { para mejora las habilidades } \\
\text { lectoras }\end{array}$ \\
\hline
\end{tabular}

Por último, la metodología seguida, Tabla 4, un 44.44\% con metodologías de: investigación-acción e investigación-participativa; con grupo de control y experimental; con aprendizaje por proyectos y rincones del aula; y con la metodología de mapas mentales. Y un $55.56 \%$ no se especifica. 


\section{RELACIÓN ENTRE NEUROCIENCIA Y PROCESOS DE ENSEÑANZA-APRENDIZAJE}

Tabla 4

Programas y Proyectos de neurociencia vinculados a la Educación: nombre y metodología

\begin{tabular}{|c|c|}
\hline Nombre & Metodología \\
\hline $\begin{array}{l}\text { Neuroeducación. Cerebro y mente en el } \\
\text { aula. Innovación y mejora de procesos } \\
\text { educativos y de enseñanza. }\end{array}$ & $\begin{array}{l}\text { Investigación- acción y investigación- } \\
\text { participativa }\end{array}$ \\
\hline $\begin{array}{l}\text { Neurociencias en Ciencias de la Educación. } \\
\text { Coordinación y formación docente desde la } \\
\text { Universidad }\end{array}$ & No se especifica \\
\hline \multicolumn{2}{|l|}{ Programa de neurociencia y educación } \\
\hline \multirow{3}{*}{$\begin{array}{l}\text { Propuestas didácticas en base a los estudios } \\
\text { neurodidácticos }\end{array}$} & Grupo control y experimental \\
\hline & $\begin{array}{l}\text { Colegio Monserrat de Barcelona: } \\
\text { Cooperación. Aprendizaje por Proyectos, } \\
\text { entre otros. }\end{array}$ \\
\hline & $\begin{array}{l}\text { Colegio Zola las Rozas de Madrid. } \\
\text { Aprendizaje en los rincones del aula } \\
\text { (atención individualizada) }\end{array}$ \\
\hline Neurodidáctica del juego & No se especifica \\
\hline $\begin{array}{l}\text { Implementación de la neurodidáctica en el } \\
\text { aula }\end{array}$ & No se especifica \\
\hline $\begin{array}{l}\text { La influencia del sueño y horario de las } \\
\text { sesiones }\end{array}$ & No se especifica \\
\hline $\begin{array}{l}\text { La Efectividad de la metodología de los } \\
\text { mapas mentales }\end{array}$ & $\begin{array}{l}\text { Metodologia de mapa mental (Grupo } \\
\text { control y experimental) }\end{array}$ \\
\hline $\begin{array}{l}\text { Influence of eye movements, auditory } \\
\text { perception and phonemic awareness in the } \\
\text { Reading process }\end{array}$ & No se especifica \\
\hline
\end{tabular}

\section{CONCLUSIONES}

Después de realizar esta revisión podemos concluir que la neurociencia se enmarca dentro de una ciencia que intenta comprender el funcionamiento y la estructura del cerebro humano (Mora y Sanguinetti, 1994, citado en Barrios- Tao, 2016) y que de ahí surge la subdisciplina de la neuroeducación, que pretende converger tres áreas: neurociencia, psicología y educación (Marina, 2012).

A lo largo de esta revisión ha estado patente el énfasis de diversos autores sobre la importancia de llevar los resultados de la neurociencia al aula (Mora, 2013, citado en Luque, 2015), aunque otros autores no compartan el mismo pensamiento. Se remarca también la importancia de la formación del docente y el cambio de rol por parte del mismo, para que implemente en su práctica educativa estrategias vinculadas con la neurociencia (Carballo, 2016; Escorza, 2017; Nizama, 2015; Pizano, 2010).

Por otra parte, se ha encontrado en las bases de datos analizadas escasos programas y proyectos vinculando neurociencia y educación en España, todavía siguen vigentes teorías cognitivas que no se vinculan con la neurociencia. Tampoco se ha encontrado una vinculación entre técnicas de estudio y neurociencia explícitamente, sin embargo, se podría trabajar para favorecer un ambiente adecuado de estudio, lo que llevaría a potenciar aún más la actividad cerebral y, por tanto, el cerebro crecerá de forma exponencial, aumentando más aún sus redes neuronales. 


\section{REFERENCIAS BIBLIOGRÁFICAS}

Aguilar, L.A., Caballero, S., Ormea, V., Aquino, R., Yaya, E., Portugal, A., Gomez, J., Zavaleta, J., y Muñoz, A. (2017). Neurociencia del sueño: rol en los procesos de aprendizaje y calidad de vida/ Neuroscience of sleep: role in learning processes and quality of life. Apuntes de ciencia \& sociedad, 7 (2), 103-109. doi: 10.18259 /acs.2017015

Barrios-Tao, H. (2016). Neurociencias, educación y entorno sociocultural. Educ. Educ., 19(3), 395-415. doi: 10.5294/edu.2016.19.3.5

Benarós, S., Lipina, S.J., Segretin, M.S., Hermida, M.J., y Colombo, J.A. (2010). Neurociencia y educación: hacia la construcción de puentes interactivos. Revista de Neurología, 50 (3), 179-186. Recuperado d e : https://dialnet.unirioja.es/servlet/articulo?codigo=4124867

Bodero, C.N. (2017). La neurociencia en la primera infancia/ Neuroscience in early childhood. Apuntes de ciencia \& sociedad, 7(1), 6-10. Recuperado de: https://dialnet.unirioja.es/servlet/articulo?codigo=6276665

Bravo, L. (2014). Neuroscience and education: current state of research on dislexia/Neurociencias y educación: estado actual de la investigación en dislexias. Estudios de Psicología/Studies in Psychology, 35, 1-28. doi: 10.1080/02109395.2014.893648

Cantó, J. (2015). Resultados de la implementación de la neurodidáctica en las aulas de educación infantil. Opción, 5, 189-199. Recuperado de:_http://www.redalyc.org/html/310/31045570011/

Carballo, A. (2016). Neuroeducación: de la neurociencia al aula. Integración. Revista sobre ceguera y deficiencia visual, (70), 1-7. Recuperado de: https://dialnet.unirioja.es/servlet/articulo?codigo=6030956

Carreiras, M. (2012). Lectura dislexia: un viaje desde Neurociencia a la Educación. Participación Educativa, 1 (1), 19-27. Recuperado de: https://dialnet.unirioja.es/servlet/articulo?codigo=4945178

Codina, M.J. (2014a). Neuroeducación en virtudes cordiales. Una propuesta a partir de la neuroeducación y la ética discursiva cordial. (Tesis doctoral). Universidad de Valencia, Valencia.

Codina, M.J. (2014b). Neuroeducación: reflexiones sobre neurociencia, filosofía y educación. Postconvencionales: ética, universidad, democracia, 7-8, 164-181. Recuperado de: https://dialnet.unirioja.es/servlet/articulo?codigo=4807471

Colegio Trinitarios (2014). Proyecto de Neurociencia- Educación Infantil. Recuperado de: http://www.alcorcon.colegiostrinitarios.com/story/proyecto-neurociencia-educaci-n- infantil

Elgier, Á. (2012). La pizarra de babel. Puentes entre neurociencia, psicología y educación. PSIENCIA. Revista Latinoamericana de ciencia psicológica, 4 (2), 149-150. doi: 10.5872/psiencia/4.2.81

Escorza, J. (2017). Crear puentes entre neurociencia y educación. Contextos: Revista de humanidades y ciencias sociales, 37, 89-96. Recuperado de: https://dialnet.unirioja.es/servlet/articulo?codigo=6152950

Guirado, I. (2017). La Neurodidáctica: Una nueva perspectiva de los procesos de Enseñanza- Aprendizaje. (Trabajo Fin de Grado). Facultad de Ciencias de la Educación, Málaga. Recuperado de: https://hdl.handle.net/10630/15524

Jauset- Berrocal, J.A., Martínez, I., y Añaños, E. (2017). Aprendizaje musical y educación: aportaciones desde la neurociencia. Cultura y Educación: Culture and Education, 29 (4), 833-847. doi: $10.1080 / 11356405.2017 .1370817$

López- Escribano, C. (2009). Aportaciones de la neurociencia al aprendizaje y tratamiento educativo de la lectura. Aula: Revista de Pedagogía de la Universidad de Salamanca, (15), 47-78. Recuperado de: https://dialnet.unirioja.es/servlet/articulo?codigo=3094484

López-Escribano, C., y Moreno, A. (2014). Neuroscience and education: developmental study of a hemispherectomy case / Neurociencia y educación: estudio evolutivo de un caso de hemisferectomía. Infancia y Aprendizaje, 37(3), 530-568. doi: 10.1080/02103702.2014.957536

Luque, M.J. (2015). Neuroeducación. Cerebro y mente en el aula. Innovación y mejora de procesos educativos y de enseñanza. En J. Gómez-Galán, E. López-Meneses, y L. Molina (eds.), Research foundations of the social 


\section{RELACIÓN ENTRE NEUROCIENCIA Y PROCESOS DE ENSEÑANZA-APRENDIZAJE}

sciences (19-26). San Juan, Puerto Rico: UMET Press.

Luque, M.J., y García, M.C. (2017). Neurociencias en Ciencias de la Educación. Coordinación y formación docente desde la universidad. En Edunovatic. Edunovatic2017 /I Congreso Virtual Internacional de Educación, Innovación y TIC. Congreso llevado a cabo en Edunovatic, online.

Marina, J.A. (2012). Neurociencia y Educación. Participación Educativa, 11(11), 7-14. Recuperado de: https://dialnet.unirioja.es/servlet/articulo?codigo=4945118

Ministerio de Educación, Cultura y Deporte (2018). Congreso de Educación Infantil y Neurociencia. Recuperado de: https://www.mecd.gob.es/educacion/mc/neurociencia- educativa/formacion/jornadas-congresos/neuroedip2018.html

Mokhtar, F., Jiménez, M.A., Heppell, S., y Segovia, N. (2016). Creando espacios de aprendizaje con los alumnos para el tercer milenio. BORDÓN. Revista de pedagogía, 68(1),61-82. Recuperado de: https://dialnet.unirioja.es/servlet/articulo?codigo $=5304655$

Nizama, M.E. (2015). Niveles de conocimiento sobre neurociencia y su aplicación en los procesos educativos. Crescendo, Institucional, 6(2), 104-113. Recuperado de: https://dialnet.unirioja.es/servlet/articulo?codigo=5294091

Pizano, G. (2010). Influencia de la neurociencia y los siete saberes en el proceso enseñanza aprendizaje y el rendimiento académico. Investigación educativa, 14(26), 21-37. Recuperado de: http://revistasinvestigacion.unmsm.edu.pe/index.php/educa/article/view/4178

Rodrigo, M. J. (2010). Where developmental psychology and neuroscience meet: A threatening or a felicitous encounter? Infancia Y Aprendizaje, 33, 3-17. doi:10.1174/ 021037010790317252

Yamila, D., De la Barrera, M.L., y Travaglia, P. (2017). Diseñar la clase. Aportes desde las neurociencias y la psicología educacional. Revista de Psicopedagogía, 34 (105), 268- 275. Recuperado de: http://pepsic.bvsalud.org/pdf/psicoped/v34n105/04.pdf

\section{FINANCIACIÓN}

Este estudio está financiado por una Beca de Iniciación a la Investigación para Estudiantes de Másteres Oficiales del Plan Propio de Investigación y Transferencia 2018 otorgada a Marta García Jiménez con N.I.F. №76626510D bajo la dirección de la profesora María Fernández Cabezas en el Departamento de Psicología Evolutiva y de la Educación de la Universidad de Granada. 